\title{
Different DNA methylome, transcriptome and histological features in uterine fibroids with and without MED12 mutations
}

Ryo Maekawa ( $\sim$ rmaekawa@yamaguchi-u.ac.jp)

Yamaguchi University Graduate School of Medicine

Shun Sato

Yamaguchi University Graduate School of Medicine

Tetsuro Tamehisa

Yamaguchi University Graduate School of Medicine

Takahiro Sakai

Yamaguchi University Graduate School of Medicine

Takuya Kajimura

Yamaguchi University Graduate School of Medicine

Kotaro Sueoka

Yamaguchi University Graduate School of Medicine

Norihiro Sugino

Yamaguchi University Graduate School of Medicine

\section{Research Article}

Keywords:

Posted Date: November 11th, 2021

DOI: https://doi.org/10.21203/rs.3.rs-1041773/v1

License: (c) (i) This work is licensed under a Creative Commons Attribution 4.0 International License.

Read Full License 
1 Different DNA methylome, transcriptome and histological features in uterine fibroids with and

without MED12 mutations

3

4 Ryo Maekawa*, Department of Obstetrics and Gynecology, Yamaguchi University Graduate School of

5 Medicine, Ube, 755-8505 Japan

6 Shun Sato, Department of Obstetrics and Gynecology, Yamaguchi University Graduate School of Medicine,

$7 \quad$ Ube, 755-8505 Japan

8 Tetsuro Tamehisa, Department of Obstetrics and Gynecology, Yamaguchi University Graduate School of

9 Medicine, Ube, 755-8505 Japan

10 Takahiro Sakai, Department of Obstetrics and Gynecology, Yamaguchi University Graduate School of

11 Medicine, Ube, 755-8505 Japan

12 Takuya Kajimura, Department of Obstetrics and Gynecology, Yamaguchi University Graduate School of

13 Medicine, Ube, 755-8505 Japan

14 Kotaro Sueoka, Department of Obstetrics and Gynecology, Yamaguchi University Graduate School of

15 Medicine, Ube, 755-8505 Japan

16 Norihiro Sugino, Department of Obstetrics and Gynecology, Yamaguchi University Graduate School of

17 Medicine, Ube, 755-8505 Japan

18

19 
20 Corresponding author

21 Ryo Maekawa, M.D., Ph. D.*

22 Department of Obstetrics and Gynecology, Yamaguchi University Graduate School of Medicine, Ube, 755-

23 8505 Japan

24 Phone : (+81)-836-22-2288

25 Fax : (+81)-836-22-2287

26 E-mail: rmaekawa@yamaguchi-u.ac.jp 
28 Abstract

29 Background: Somatic mutations in Mediator complex subunit 12 (MED12m) have been reported as a

30 biomarker of uterine fibroids (UFs). However, the role of MED12m is still unclear in the pathogenesis of

31 UFs. Therefore, we investigated the differences in DNA methylome, transcriptome, and histological

32 features between MED12m-positive and -negative UFs.

33 Methods: DNA methylomes and transcriptomes were obtained from MED12m-positive and -negative UFs

34 and myometrium, and hierarchically clustered. Differentially expressed genes in comparison with the

myometrium and co-expressed genes detected by weighted gene co-expression network analysis were subjected to gene ontology enrichment analyses. The amounts of collagen fibers and the number of blood vessels and smooth muscle cells were histologically evaluated.

Results: Hierarchical clustering based on DNA methylation clearly separated the myometrium, MED12mpositive, and MED12m-negative UFs. MED12m-positive UFs had the increased activities of extracellular matrix formation, whereas MED12m-negative UFs had the increased angiogenic activities and smooth muscle cell proliferation.

Conclusion: The MED12m-positive and -negative UFs had different DNA methylation, gene expression, and histological features. The MED12m-positive UFs form the tumor with a rich extracellular matrix and mutations affect the tissue composition of UFs. 
Introduction

Uterine fibroids are tumors derived from uterine smooth muscle cells and are most common in

gynecologic neoplasms ${ }^{1}$. In the last decade, somatic mutations of Mediator complex subunit 12 (MED12)

have been found to be reliable biomarkers of uterine fibroids ${ }^{2-4}$. MED12 is located on the X chromosome

and encodes the RNA polymerase II mediator complex and part of the transcriptional preinitiation machinery.

Mutations of MED12, especially mutations in exon 2, are thought to be the underlying causes of about $70 \%$

of human uterine fibroids ${ }^{2,5,6}$. However, the remaining $30 \%$ of uterine fibroids do not have MED12

mutations, which indicates that the role of MED12 mutations in the pathogenesis of uterine fibroids is unclear.

mutations are reported to be smaller and often more numerous than those without MED12 mutations ${ }^{4}$.

Several reports have suggested links between MED12 mutations and different phenotypes of uterine fibroids.

Uterine fibroids without MED12 mutations were found to have elevated erythropoietin expression in an

estrogen-dependent manner, while the uterine fibroids with MED12 mutation had low erythropoietin ${ }^{7,8}$.

Furthermore, uterine fibroids with and without MED12 mutations had different cell components and

different amounts of collagen ${ }^{9}$. These reports suggest that MED12 mutations are associated with different

phenotypes of uterine fibroids. On the other hand, genome-wide gene expression profiles were not 
fibroid phenotypes are associated with MED12 mutations.

DNA methylation is a major type of epigenetic mark. DNA methylation profiles define each type

of normal cells and distinguish cell types ${ }^{12-14}$, and therefore have been used to characterize abnormal cells

13,14. DNA methylation is tissue/cell-specific, and DNA methylation profiling is more useful than profiling mRNA expression to define the cell identity ${ }^{15}$. We previously reported that uterine fibroids had different DNA methylation profiles from normal myometrium by genome-wide approach and that DNA methylation profiles segregated the uterine fibroids and normal myometrium ${ }^{15,16}$. Furthermore, using these differently methylated genes between uterine fibroids and normal myometrium, we found some potential mechanisms for the pathogenesis of uterine fibroids ${ }^{15-19}$. We also found significant differences in DNA methylation levels of those genes between uterine fibroids with and without MED12 mutations ${ }^{16}$. These findings led us to investigate differences in genome-wide DNA methylation profiles between uterine fibroids with and without MED12 mutations.

We recently identified SATB2 and NRG1 as potential upstream regulatory factors in uterine fibroids ${ }^{19}$. SATB2 and NRG1 expressions were increased in uterine fibroids compared to the myometrium. Both SATB2 and NRG1 activated WNT/beta-catenin and TGF-beta signaling pathways, which are related to the pathogenesis of uterine leiomyomas ${ }^{19}$. Interestingly, established cell lines overexpressing SATB2 morphologically changed from spindle-like forms to fibroblast-like forms with elongated protrusions ${ }^{19}$, suggesting that SATB2 and NRG2 play essential roles in initiating tumorigenesis in uterine fibroids. However, the association between the expression of NRG1 and SATB2, and MED12 mutations is still unclear. 
uterine fibroids: 1) genome wide methylation profiles, 2) cellular functions as revealed by transcriptome analyses, 3) different histopathological features and 4) the expressions of NRG1 and SATB2.

\section{Results}

Hierarchical clustering using DNA methylation profiles

We first examined the DNA methylome of the uterine fibroids with and without MED12 mutations

(MED12m-positive uterine fibroids $(n=6)$ and MED12m-negative uterine fibroids $(n=12)$, respectively), uterine fibroids (Fig. 1a). The MED12m-positive and -negative uterine fibroids were classified into different clusters (Fig. 1a), suggesting that uterine fibroids with and without MED12 mutations are different at the molecular levels. fibroids can be further classified into three clusters (Subtype-1, -2, and -3; Fig. 1a). Subtype-1 was classified into the same cluster as the MED12m-positive uterine fibroids (Fig. 1a). Subtypes-2 and -3 were classified into clusters different from Subtype-1 (Fig. 1a). Figure $1 \mathrm{~b}$ shows the distribution of aberrantly methylated CpGs in the MED12m-positive and negative uterine fibroids compared to the myometrium throughout the chromosomes. The results showed that the DNA methylation statuses of Subtype-1 are similar to that of the MED12m-positive uterine fibroids. 
103 On the other hand, in Subtypes-2 and -3 , the DNA methylation status in the autosomes tended to be

104 hypermethylated compared to that in Subtype-1 and the MED12-positive uterine fibroids (Fig. 1a).

Differentially expressed genes (DEGs)

We determined DEGs in the MED12m-positive and -negative uterine fibroids compared to the

myometrium. The MED12m-positive fibroids had 157 increased and 233 decreased genes compared to the

myometrium (Supplemental Tables S1 and S2 online). The MED12m-negative fibroid had 110 increased and 207 decreased genes compared to the myometrium (Supplemental Tables S3 and S4 online). The DEGs

111 were subjected to the GO enrichment analysis to know the characteristics of the DEGs.

The GO terms "telomere organization", "DNA replication-dependent nucleosome assembly",

113 "positive regulation of gene expression epigenetic", "extracellular matrix organization", "collagen catabolic

114 process", "cell adhesion", "integrin-mediated signaling pathway", "cellular protein metabolic process",

115 "response to estrogen", and "canonical Wnt signaling pathway" were detected in the increased genes in the

116 MED12m-positive uterine fibroids (Fig. 2a). In the decreased genes in the MED12m-positive uterine

117 fibroids (Fig. 2b), the GO terms "reactive oxygen species metabolic process", "inflammatory response",

118 "regulation of inflammatory response", "angiogenesis", "regulation of macrophage activation", and "positive

119 regulation of apoptotic process" were detected.

In the MED12m-negative uterine fibroids, the GO terms "cellular protein metabolic process", 
expression epigenetic", "liver regeneration", and "negative regulation of canonical Wnt signaling pathway"

were detected in the increased genes (Fig. 2d). In the decreased genes in the MED12m-negative uterine

124 fibroids (Fig. 2d), the GO terms "cell adhesion", "extracellular matrix organization", "positive regulation of cell-substrate adhesion", "integrin-mediated signaling pathway", "negative regulation of transcription by

RNA polymerase II", "inflammatory response", "reactive oxygen species metabolic process", "positive regulation of apoptotic process", and "transforming growth factor-beta receptor signaling pathway" were detected.

Figure 2e summarizes the results of GO enrichment analyses of DEGs. Compared to myometrium, MED12m-positive uterine fibroids showed increased activities of extracellular matrix organization, cell adhesion, integrin-mediated signaling, and Wnt signaling pathway, whereas MED12mnegative uterine fibroids had the decreased activities of them and TGF-beta signaling, suggesting that MED12m-positive uterine fibroids have the increased activity of extracellular matrix formation compared increased responsiveness to estrogen and decreased angiogenic activities. Both types of uterine fibroids had increased cell proliferation and transcription activities and decreased inflammatory response and

137 reactive oxygen species metabolic process activities compared to myometrium. Figure 3 shows the processes between the MED12m-positive and MED12m-negative uterine fibroids. 
141

142

143

The preceding DEGs (Fig. 2) is based on comparing the uterine fibroids with the myometrium,

and this analytic approach has been used so far ${ }^{10,11}$. In general, in the analytic method that compares the

target tissues to the control tissues, there is a possibility of missing the essential character of the target tissue

when the cell character of the target tissue is close to that of the control tissue. Therefore, to know the intrinsic character in each of the MED12m-positive and -negative uterine fibroids, we used a WGCNA

analysis ${ }^{20,21}$. WGCNA is a system biology method to describe the correlation patterns among the genes across microarray samples such as transcriptome data and to find the groups with highly correlated genes that work in the same biological functions ${ }^{20,21}$. We defined groups consisting of highly correlated genes as co-expressed gene (COG) groups and detected unique properties in the MED12m-positive and -negative uterine fibroids by comparing the intrinsic functions of each tissue.

The transcriptome data of the MED12m-positive and -negative uterine fibroids were independently subjected to the WGCNA. In the MED12m-positive and -negative uterine fibroids, WGCNA identified 26 and 14 COG groups, respectively (Table 1), and these genes were subjected to the GO enrichment analysis.

In the MED12m-positive uterine fibroids, three of the 26 COG groups had significant GO terms, while in the MED12m-negative uterine fibroids, five of the 14 COGs groups had significant GO terms (Table 1). Figure 4 shows the specific GO terms from three COG groups in the MED12m-positive (Group1, Group2, and Group3) and five COG groups in the MED12m-negative (Group1, Group2, Group3, Group4, 
160 and Group5), respectively. The commonly detected GO terms between the MED12m-positive and -

161 negative uterine fibroids included "RNA splicing, via transesterification reactions", "mRNA splicing, via

162 spliceosome", ncRNA processing", "mRNA processing", and "RNA splicing", which are related to

163 transcription and translation, and "ribonucleoprotein complex biogenesis" and "DNA replication", which are

164 related to cell proliferation (Fig. 4).

The extracellular matrix-related terms including "extracellular structure organization" and

"extracellular matrix organization" were also commonly found. The gene ratios were much larger in the

MED12m-positive uterine fibroids than those in the MED12m-negative uterine fibroids, suggesting that the number of extracellular matrix-related genes was larger in the MED12m-positive uterine fibroids than that in the MED12-negative uterine fibroids (Fig. 4).

We then focused on specific GO terms in each of the MED12m-positive or -negative uterine

fibroids. There were no specific GO terms to the MED12m-positive uterine fibroids (Fig. 4). On the

173 ("Golgi vesicle transport", "ER to Golgi vesicle-mediated transport", and "Rho protein signal transduction"), angiogenesis") (Fig. 4). 
of extracellular matrix organization compared with MED12m-negative uterine fibroids. On the other hand,

MED12m-negative uterine fibroids showed increased activities of angiogenesis and smooth muscle cell

proliferation. Both types of uterine fibroids had increased activities of cell proliferation and transcription for gene expression.

Immunofluorescence staining

uterine fibroids have increased activities of extracellular matrix organization and that the MED12m-negative

and 4). We histologically examined the amount of collagen fibers in the MED12m-positive and -negative 
was significantly higher in the MED12m-negative uterine fibroids than the MED12m-positive uterine

fibroids and myometrium (Fig. 5e and 5f). There was no significant difference between the MED12m-

positive uterine fibroids and myometrium.

Upstream regulators in uterine fibroids

To know whether mutations in MED12 are associated with the upregulation of upstream regulators,

SATB2 and NRG1 in uterine fibroids, we examined the DNA methylation and expression levels of SATB2

and NRG1 in the uterine fibroids with and without MED12 mutations. In SATB2, 88.9\% (8 of 9 samples)

of the MED12m-positive uterine fibroids and 75\% (9 of 12 samples) of the MED12m-negative uterine

fibroids showed higher DNA methylation levels (more than 15\% DNA methylation) than the myometrium,

MED12m-positive uterine fibroids and 75\% (9 of 12 samples) of the MED12m -negative uterine fibroids

S5 online).

213 (9 of 12 samples) of the MED12m-negative uterine fibroids were more than twice those in the myometrium

214 (Fig. 6c and Supplemental Table S5 online), while the mRNA expression levels of NRG1 in all the

215 MED12m-positive uterine fibroids and 67\% (8 of 12 samples) of the MED12m-negative uterine fibroids 
217 methylation and mRNA expression of at least one of SATB2 and NRG1 were higher in all the MED12m-

218 positive and -negative uterine fibroids than they were in the myometrium. Since DNA hypermethylation

219 and increased expression of SATB2 or NRG1 were observed regardless of MED mutations, these

220 characteristics are unlikely to depend on MED12 mutations.

\section{Discussion}

The present study showed that the DNA methylation profiles of the MED12m-positive and -negative

uterine fibroids differed. Since DNA methylation is cell/tissue-specific, uterine fibroids with MED12

mutations differ from uterine fibroids without MED12 mutations at the molecular level. This prompted us

to clarify the difference between the two types of uterine fibroids in this study.

matrix organization. The WGCNA analysis also showed the activated extracellular matrix organization in

the MED12m-positive uterine fibroids. On the other hand, in the MED12m-negative uterine fibroids, most

of the genes in the GO terms related to extracellular matrix organization were down-regulated in comparison

231 with the myometrium and MED12m-positive uterine fibroids. Furthermore, the TGF-beta signaling

232 pathway, which contributes to fibrosis ${ }^{22,23}$, was downregulated in the MED12m-negative uterine fibroids.

233 These results suggest that MED12 mutations activate extracellular matrix organization in uterine fibroids.

234 In fact, our histological results showed that the amount of collagen was enriched in the uterine fibroids with 
contribute to collagen synthesis, and increased collagen deposition in uterine fibroids ${ }^{11,24}$. Also, many of the uterine fibroids in those studies may have been MED12m-positive because more than $70 \%$ of uterine fibroids are MED12m-positive. to muscles. In our histological results, the amount of smooth muscle cells was larger in the uterine fibroids without MED12 mutations compared with the uterine fibroids with MED12 mutations, which is consistent with a previous report demonstrating a high ratio of smooth muscle cells to fibroblasts in the uterine fibroids without MED12 mutations compared to that with MED12 mutations ${ }^{9}$. That study, together with the present results suggest that uterine fibroids without MED12 mutations are enriched in smooth muscle cells and contain a low amount of collagen fibers, and that MED12 mutations are associated with collagen-rich uterine fibroids. well reflects one of the characters of uterine fibroids, which is the activated cell proliferation of smooth muscle cells or fibroblasts. It is interesting to note that the activity of the Wnt signaling pathway was

251 decreased in MED12m-negative uterine fibroids. The Wnt signaling pathway has been reported to play an included in those reports was MED12m-positive uterine fibroids in which Wnt signaling pathway is activated. 
255 than the Wnt signaling pathway. As shown in Table 2, multiple signaling pathways are involved in cell

256 proliferation in both MED12m-positive and -negative uterine fibroids.

VEGF expression is reported to be upregulated in uterine fibroids ${ }^{25}$, which suggests that

angiogenic activity in increased in uterine fibroids. On the other hand, our GO enrichment analysis

suggested that angiogenesis is downregulated in MED12m-positive uterine fibroids and upregulated in

MED12m-negative uterine fibroids. There seems to be a discrepancy between the previous reports and our

results. That may be due to the difference in cellular components of the tissue samples of uterine fibroids.

As shown in the histological features of both types of uterine fibroids, MED12m-positive uterine fibroids

MED12m-positive uterine fibroids.

fibroids. Since fibroblasts were reported to proliferate or produce collagen in response to estrogen while

fibroids seem to well respond to estrogen. processes are decreased in both MED12m-positive and -negative uterine fibroids. That is not surprising 
reactive oxygen species 252627,28 . uterine fibroids carried both the MED12 mutation and HMGA2 overexpression, and that only half of the also indicated that the MED12 mutations and increased HMGA2 expression co-existed in the same uterine fibroid nodule ${ }^{10,31}$. pathogenesis of uterine fibroids ${ }^{19,22,23}$. Whether or not MED12 has a mutation, SATB2 and NRG1 were more strongly expressed in uterine fibroids than in the myometrium, which indicates that the upregulation of NRG1 and SATB2 are independent of MED12 mutations. Our results also indicated that all the expression in either SATB2 or NRG1, suggesting that the dysregulation of upstream regulatory factors such as SATB2 and NRG1 is involved in the pathogenesis of uterine fibroids. 
293 differences among the three subtypes of uterine fibroids without MED12 mutation.

295 clearly differ in DNA methylation, gene expression, and histological features. The DNA methylome

296 indicated that the uterine fibroids carrying MED12 mutations differed from the uterine fibroids without

297 MED12 mutations, and that MED12 mutations do not directly change DNA methylation profiles of uterine

298 fibroids. The transcriptome and histological examination revealed that the MED12m-positive uterine

299 fibroids increased extracellular matrix production activity compared with the MED12m-negative uterine

300 fibroids. MED12 mutations may affect the phenotypes of uterine fibroids by modulating the production of

301 extracellular matrix. Both types of uterine fibroids had increased cell proliferation activities, but they may

302 use different signaling pathways for growth. The present study shows that uterine fibroids differ depending

303 on the presence of MED12 mutations.

Methods

Ethics Statement

Graduate School of Medicine. Written informed consent was obtained from the participants before 
Tissues of uterine fibroid and myometrium were obtained from 42 Japanese women, respectively.

314 Uterine fibroids were obtained from patients aged 33-45 who underwent hysterectomy for uterine fibroids.

315 Myometrium was obtained from patients with uterine fibroids aged 34-42 who underwent hysterectomy for

316 uterine fibroids or early stage of cervical cancer. None of the women enrolled in this study received

317 previous treatment with sex steroid hormones or gonadotropin-releasing hormone agonists/antagonists.

318 We analyzed the uterine fibroids with MED12 mutation status by Sanger sequencing as reported previously

319 (Supplemental Fig. S1 online) ${ }^{16}$. All of the MED12m-positive uterine fibroids had the point mutations in

MED12 gene. MED12 expression levels were not significantly different between myometrium and uterine

fibroids with or without MED12 mutation.

HMGA2 rearrangements are thought to be one of the mutations driving the development of uterine rearrangements oocur with the highest frequency ${ }^{3,30}$. More than $80 \%$ of uterine fibroids possess karyotypic abnormalities, and MED12 mutations and HMGA2 rearrangements encompass approximately $80-90 \%$ of genetic alterations in uterine fibroids ${ }^{3,29,30}$. Previous reports indicated that the MED12 mutations and 
330 the MED12 mutations and HMGA2 rearrangements were alternatively associated with the pathogenesis of

331 uterine fibroids. Hence, past reports compared the features between the uterine fibroids with the MED12

332 mutations and that with HMGA2 rearrangements ${ }^{9,10}$. The uterine fibroids carrying HMGA2

333 rearrangement are reported to overexpress HMGA2 ${ }^{10}$. To investigate whether our samples included the

334 uterine fibroids carrying HMGA2 rearrangement, we examined the expression levels of HMGA2 in the

335 MED12m-negative and MED12m-positive uterine fibroids, and myometrium using transcriptome analyses.

336 Three of the nine MED12m-negative uterine fibroids had expressions more than two-fold of the mean

expression in the myometrium (Supplemental Fig. S2 online). Moreover, one of the six MED12m-positive

uterine fibroids had expressions more than two-fold of the mean expression in the myometrium

339 (Supplemental Fig. S2 online). These results suggest that 1) a number of the MED12m-negative uterine

fibroids lack HMGA2 rearrangements, and 2) MED12 mutations and HMGA2 rearrangements can co-exist.

These facts led us to compare the uterine fibroids with and without MED12 mutations rather than to compare

uterine fibroids with MED12 mutations and HMGA2 rearrangements.

346 DNA kit (Qiagen, Valencia, CA, USA), as previously reported ${ }^{32}$. DNA methylation was analyzed with an

347 Illumina Infinium assay with the HumanMethylation450 BeadChip (Illumina, San Diego, CA, USA), which 
to 3'-UTR of consensus coding sequences. Methylated and unmethylated signals were used to compute

beta-values, which are quantitative scores of the DNA methylation levels, ranging from 0 (completely

(Illumina) according to the manufacturer's instructions. CpGs with "detection $p$ values" $>0.01$ (computed

from the background based on negative controls), CpGs that were zero in all samples, and CpGs on Y

Database (https://www.ncbi.nlm.nih.gov/refseq/) as reference genes.

Transcriptome analysis

analyzed as previously reported ${ }^{33,34}$. Total RNAs were isolated from cells by using an RNeasy mini kit

361 (Qiagen). Target cDNA for a microarray was prepared from 250 ng of total RNA with the Ambion WT

362 Expression kit (Ambion, Austin, TX, USA) and the GeneChip WT PLUS reagent kit (Affymetrix).

363 Transcriptomes were analyzed with a GeneChip Human Genome 1.0 ST Array (Affymetrix, Santa Clara,

364 CA, USA) as previously reported ${ }^{33,34}$. The microarray was spotted with 21,014 RefSeq genes.

365 Hybridization to the microarrays, washing, staining, and scanning was performed using the GeneChip

366 system (Affymetrix) composed of the Scanner 30007 G Workstation Fluidics 450 and the Hybridization

367 Oven 645. The scanned image data were processed using a gene expression analysis with the Patrek 
368 Genomics Suite 6.5 software program (Partech, Munster, Germany). All expression data were converted

369 to $\log 2$ values. Differentially expressed genes (DEGs) were extracted when the expressions in the

370 MED12m-positive or -negative uterine fibroids were higher than 2.0-fold or less than 0.5 -fold of that in the

371 myometrium, and $\mathrm{p}<0.05$ (t-test), and the average expression levels in the tissues with higher expression

372 were more than 100 .

Weighted gene co-expression network analysis (WGCNA)

We employed a weighted gene co-expression network analysis (WGCNA) package in $\mathrm{R}^{20,21}$ according to the manufacturer's instructions to identify genes that were co-expressed in uterine fibroids and myometrium. Low signal probes with values $<10$ in more than $90 \%$ of the samples were considered as noise and removed, and correlations based on mostly zero counts are not meaningful. To stabilize the samples' variance, we used the variance stabilizing transformation function in DESeq2 ${ }^{35}$. The blockwiseModule function was used with the parameters; power $=12$; minimum module size $=50$; deep split $=0$; cut height $=0.95$; gene group merge height $=0.25$. Detected gene groups were subjected to gene annotation 

of paraffin-embedded samples were deparaffinized, washed with cold phosphate-buffered saline (PBS), placed in preheated Bouin's fluid overnight, rinsed in tap water until completely clear, rinsed in distilled water, stained with Weigert's Iron Hematoxylin for 10 minutes, rinsed in tap water for 2 minutes, rinsed in distilled water, immersed in Biebrich Scarlet/Acid Fuchsin Solution for 10 minutes, rinsed in distilled water, defferentiated in Phosphomolybdic/Phosphotungstic Acid Solution for 15 minutes, placed in Aniline Blue Solution for 15 minutes, rinsed in distilled water, immersed in Acetic Acid Solution (1\%) for 1 minute, dried, placed in Xylene 3 times and mounted in synthetic resin. The area of collagen fibers, which were stained blue, was quantified by Image $J$, and the percentage per field of view was calculated. The calculations area $(\%)$ in each tissue. phosphate-buffered saline (PBS), and blocked with blocking solution (10\% bovine fetal serum and $1 \%$ bovine serum albumin in PBST) for $60 \mathrm{~min}$. Then the cells were incubated with mouse anti- $\alpha \mathrm{SMA}$ monoclonal antibody for smooth muscle cell staining (Abcam, Tokyo, Japan; Cat\# ab7817, RRID: AB_262054) and rabbit anti-CD31 monoclonal antibody for vascular endothelial cells (Abcam, Cat\# ab182981, RRID: AB_2756834) as primary antibody (diluted at 1:500 in the blocking solution) at 4 C 
ab150113: RRID: AB_2576208; Abcam, Cat\# ab150116: RRID: AB_2650601) and the Alexa Fluor 594

conjugated goat anti-rabbit IgG (Abcam, Cat\# ab150084, RRID: AB_2734147) as secondary antibodies (diluted at 1:1000 in PBS) for 45 min a room temperature, respectively. The number of blood vessels,

calculated. The calculations were done on 5 randomly chosen areas at $\mathrm{x} 100$, and the average numbers were in each tissue section. considered significant.

\section{Bioinformatics}


425 Encyclopedia of Genes and Genomes; KEGG), respectively ${ }^{37}$. In GO analysis, GO terms with adjusted p

426 (BH method) $<0.01$ were considered significant enrichment. In KEGG analysis, pathways with $\mathrm{p}<0.05$

427 were considered significant enrichment. In WGCNA analysis, adjusted $\mathrm{p}<0.1$ was regarded as substantial

428 enrichment in the GO enrichment analysis. Hierarchical clustering was performed in R using the Ward

429 method ${ }^{38}$. Chromosomal distributions of the DNA methylation statuses of all CpG loci in the MED12m-

430 positive and -negative uterine fibroids compared to the myometrium were examined using "chromoMap"

431 implemented in $\mathrm{R}$ (https://cran.r-project.org/web/packages/chromoMap/index.html). CpG sites, which

432 have $\mathrm{p}<0.05$ and beta-value difference $>0.2$ compared to the myometrium, are plotted in autosomes and $\mathrm{X}$

433 chromosome. The GO terms were summarized by removing redundancy and plotted using reduce and

434 visualize gene ontology (REVIGO) with Allowed Similarity as "small (0.5)" 39.

436 Combined Bisulfite Restriction Analysis (COBRA)

DNA methylation levels were evaluated by COBRA as we previously reported ${ }^{15,16}$. In brief,

sodium bisulfite treatment was performed using an EpiTect Bisulfite kit (Qiagen) according to the conditions

as follows: $95{ }^{\circ} \mathrm{C}$ for $5 \mathrm{~min}, 65^{\circ} \mathrm{C}$ for $85 \mathrm{~min}, 95{ }^{\circ} \mathrm{C}$ for $5 \mathrm{~min}$ and $65{ }^{\circ} \mathrm{C}$ for $175 \mathrm{~min}$. After sodium

440 bisulfite treatment, PCR was performed using one unit of Biotaq HS DNA polymerase (Bioline, London,

441 UK) and the primer sets shown in Supplemental Table S6 online under the thermocycling conditions (35 to

44238 cycles of $95^{\circ} \mathrm{C}$ for $30 \mathrm{sec}, 60^{\circ} \mathrm{C}$ for $30 \mathrm{sec}$, and $72{ }^{\circ} \mathrm{C}$ for $30 \mathrm{sec}$, with an initial step of $95{ }^{\circ} \mathrm{C}$ for $10 \mathrm{~min}$ 
444 TaqI (Takara, Tokyo, Japan) or HpyCH4IV (New England Biolabs, Ipswich, MA). The treated PCR

445 product was electrophoresed by $3 \%$ agarose gel. PCR products from methylated DNA and unmethylated

446 DNA are digested and undigested by the treatment with the restriction enzyme. The intensity of the signals

447 of the digested and undigested PCR products was measured by densitometry. Methylation levels (\%) were

448 calculated as the ratio of the digested PCR product in the total PCR product (digested + undigested products).

Quantitative real-time RT-PCR (qRT-PCR)

451

Total RNA was isolated from tissues and cells using Isogen (Wako Pure Chemical Industries Ltd,

452

Osaka, Japan). One $\mu$ g total RNA was reverse-transcribed using a Quantitect Reverse Transcription Kit

453 (Qiagen) according to the manufacturer's protocol as previously reported ${ }^{15}$. A primer pair for

454 glyceraldehyde-3-phosphate dehydrogenase $(G A P D H)$ was used as an internal control. Real-time qRT-

455 PCR was performed using SYBR Premix Ex Taq (Takara, Ohtsu, Japan) and a LightCycler (Roche Applied

456 Science, Basel, Switzerland). All samples were run in duplicate. The relative quantity of cDNA was

457 calculated with the $\Delta \Delta \mathrm{Ct}$ method. Melting curves of the products were obtained after cycling by a stepwise

Supplemental Table S6 online.

All statistical analyses were performed in $\mathrm{R}^{38}$. 


\section{References}

$4691 \quad$ Stewart, E. A. Uterine fibroids. Lancet 357, 293-298 (2001).

4702 Makinen, N. et al. MED12, the mediator complex subunit 12 gene, is mutated at high frequency in uterine leiomyomas. Science 334, 252-255 (2011).

4723 Mehine, M. et al. Characterization of uterine leiomyomas by whole-genome sequencing. $N$ Engl J Med 369, 43-53 (2013).

$474 \quad$ Bullerdiek, J. \& Rommel, B. Factors targeting MED12 to drive tumorigenesis? F1000Res 7, 359, doi:10.12688/f1000research.14227.2 (2018).

4765 Markowski, D. N. et al. MED12 mutations in uterine fibroids--their relationship to cytogenetic subgroups. Int J Cancer 131, 1528-1536 (2012).

4786 Mittal, P. et al. Med12 gain-of-function mutation causes leiomyomas and genomic instability. J Clin Invest 125, 3280-3284 (2015). 

Subtypes and Homeotic Transformation in Uterine Fibroids. Cell reports 29, 4069-4085.e4066, doi:10.1016/j.celrep.2019.11.077 (2019). gene expression profiling analysis. Mol Med Rep 17, 6985-6996 (2018). doi:10.1038/emm.2017.10 (2017). ovarian endometrioma. J Ovarian Res 12, 14, doi:10.1186/s13048-019-0489-1 (2019). in a T-DMR. Mol Endocrinol 30, 335-347 (2016). pathogenesis and development of uterine leiomyomas. PLoS One 8, e66632, 
$501 \quad 16 \quad$ Sato, S. et al. Identification of uterine leiomyoma-specific marker genes based on DNA methylation and their clinical application. Sci Rep 6, 30652, doi:10.1038/srep30652 (2016). on the X chromosome in uterine leiomyoma. J Reprod Dev 57, 604-612 (2011). leiomyomas. J Reprod Dev 60, 47-54 (2014). Reprod Genet 36, 2385-2397 (2019). Appl Genet Mol Biol 4, Article17, doi:10.2202/1544-6115.1128 (2005).

$51121 \quad$ Langfelder, P. \& Horvath, S. WGCNA: an R package for weighted correlation network analysis. BMC Bioinformatics 9, 559, doi:10.1186/1471-2105-9-559 (2008). 18, doi:10.3390/ijms18112435 (2017).

51724 Reis, F. M., Bloise, E. \& Ortiga-Carvalho, T. M. Hormones and pathogenesis of uterine fibroids. Best Pract Res Clin Obstet Gynaecol 34, 13-24 (2016). 
doi:10.3390/cells10050982(2021).

52126 Leppert, P. C., Catherino, W. H. \& Segars, J. H. A new hypothesis about the origin of uterine fibroids based on gene expression profiling with microarrays. Am J Obstet Gynecol 195, 415-420, (2006). Progenitor Cells. Stem Cells Int 2018, 1716246, doi:10.1155/2018/1716246 (2018). potential role of hypoxia in the pathophysiology of uterine fibroids. $J$ Assist Reprod Genet 30, 969-974, (2013). and leiomyosarcoma. Modern pathology : an official journal of the United States and Canadian Academy of Pathology, Inc 27, 1144-1153 (2014). MED12 mutation in uterine leiomyoma. Mol Hum Reprod 24, 556-563 (2018). doi:10.1186/s12864-019-5695-0 (2019). 

stromal cells. PLoS One 9, e83612, doi:10.1371/journal.pone.0083612 (2014).

54034 Mihara, Y. et al. An Integrated Genomic Approach Identifies HOXC8 as an Upstream Regulator in Ovarian Endometrioma. J Clin Endocrinol Metab 105, doi:10.1210/clinem/dgaa618 (2020). data with DESeq2. Genome biology 15, 550, doi:10.1186/s13059-014-0550-8 (2014).

54436 Yu, G., Wang, L. G., Han, Y. \& He, Q. Y. clusterProfiler: an R package for comparing biological themes among gene clusters. OMICS 16, 284-287 (2012).

54637 Huang da, W. et al. DAVID Bioinformatics Resources: expanded annotation database and novel algorithms to better extract biology from large gene lists. Nucleic Acids Res 35, W169-175, doi:10.1093/nar/gkm415 (2007).

$549 \quad 38 \quad$ R Core Team. R: A language and environment for statistical computing. (2021).

$55039 \quad$ Supek, F., Bosnjak, M., Skunca, N. \& Smuc, T. REVIGO summarizes and visualizes long lists of gene

554 This work was supported in part by JSPS KAKENHI (Grant Number 19K09803, 21K09495, 20K18222, 
561 the manuscript.

562

563 Competing interests

564 The authors declare no competing interests.

565

566

\section{Data availability}

567 The data underlying this article are available in the Dryad Digital Repository at

568 https://doi.org/10.5061/dryad.sn02v6x4d (please use the following link during review process

569 https://datadryad.org/stash/share/eyqm-aWxXAEWt2-CK7nZ4aukRX40dF8WLoz1xnw8WLc). 
Figure 1. DNA methylation profiling of the MED12m-positive and -negative uterine fibroids, and myometrium.

a. DNA methylation profiles of the MED12m-positive and -negative uterine fibroids, and myometrium were compared using hierarchical clustering analyses. Distances of DNA methylation pattern are indicated as height. Each color indicates the myometrium (light blue), the MED12m-positive uterine fibroids (red), and the MED12m-negative uterine fibroids (green). The MED12m-negative uterine fibroids were further classified into three different clusters, Subtype-1, Subtye-2, and Subtype-3. b. Chromosomal distribution of hyper- or hypomethylated CpGs in the MED12m-positive and -negative uterine fibroids (Subtype-1, -2, and -3) compared to the myometrium are shown. The locations of $\mathrm{CpG}$ sites, which have $\mathrm{p}<0.05$ and betavalue difference $>0.2$ compared to the myometrium, are indicated with red (hypermethylated CpGs) or blue (hypomethylated CpGs). Autosomal and sex chromosome numbers are shown on the top.

584 Figure 2. The scatterplot of GO terms in DEGs.

585 The plots and tables show the GO terms after the redundancy reduction in the MED12m-positive-increased

586 (a), -decreased (b), the MED12m-negative-increased (c), and -decreased (d) DEGs. The colors indicate the $587 \log 10$ (p-value) of the summarized GO terms. The size of the circle indicates the frequency of the GO term in the underlying GO database. The circles of more general terms are plotted larger. The color and the size of circles are plotted according to the default setting of REVIGO ${ }^{39}$. e. Summary of GO analysis in the MED12m-positive and -negative uterine fibroids. 
591 The specific terms to the MED12m-positive uterine fibroids, the MED12m-negative uterine fibroids, and commonly detected terms are shown. Red and blue mean higher and lower expression compared to the myometrium

594 Figure 3. Expression levels of representative genes in the detected biophysical processes.

596 MED12m-positive and -negative uterine fibroids were indicated. a, $\mathrm{p}<0.01$ (myometrium ( $\mathrm{n}=6$ ) vs.

597 MED12m-positive uterine fibroids $(\mathrm{n}=6)$ ). $\quad \mathrm{b}, \mathrm{p}<0.01$ (myometrium ( $\mathrm{n}=6)$ vs. MED12m-negative uterine 598 fibroids ( $\mathrm{n}=9))$.

Figure 4. Enriched GO terms identified by weighted gene co-expression network analysis (WGCNA) in the MED12m-positive and -negative uterine fibroids.

601 Twenty-six and 14 COGs groups in the MED12m-positive and -negative uterine fibroids, respectively, were introduced into the KEGG pathway and GO enrichment analyses. Three and five COGs groups in the MED12m-positive and -negative uterine fibroids were significantly enriched with GO terms. The other 23

604 and 9 COGs groups in the MED12m-positive and -negative uterine fibroids, respectively, were not 605 significantly enriched with GO terms. The ratio of the number of identified genes to all genes in each term 606 is shown as "geneRatio". P-values were adjusted with the BH method by clusterProfiler ${ }^{36}$ and indicated 607 with colors.

608 Figure 5. Histological examination in the uterine fibroids and myometrium.

609 a. Immunofluorescent staining for collagen fibers in the MED12m-positive, -negative uterine fibroids, and 
610 myometrium. Collagen fibers are detected as blue by a trichrome staining kit (TRM-1, ScyTec

611 Laboratories inc). b. Boxplots show the occupation rate of collagen fiber. The collagen fiber area was

612 quantified by Image J. The percentage per view field was calculated on 15 randomly chosen areas at x200

613 magnification, and average percentages were indicated in each tissue section. $*, p<0.05$. c.

614 Immunofluorescent staining for smooth muscle cells ( $\alpha$ SMA, green) and vascular endothelial cells (CD31,

615 red) in the MED12m-positive, -negative uterine fibroids, and myometrium. d. Boxplots show the number

616 of blood vessels, which was counted by Image J. The number per view field was calculated on 5 randomly

617 chosen areas at x100 magnification, and the average numbers were indicated in each tissue section. *, $\mathrm{p}<$

618 0.05. e. Immunofluorescent staining for smooth muscle cells ( $\alpha$ SMA, red) and nucleus (DAPI, blue) in the

619 MED12m-positive, -negative uterine fibroids, and myometrium. The cells stained with aSMA were

620 considered as smooth muscle cells, whereas the cells that were not stained with aSMA was considered as

621 non-smooth muscle cells. f. Boxplots show the percentage of the smooth muscle cells in the smooth and

622 non-smooth muscle cells. The number per view field was calculated on 5 randomly chosen areas at x200

623 magnification, and the average numbers were indicated in each tissue section. $\quad *, p<0.05$.

624 Figure 6. DNA methylation and mRNA expression statuses of SATB2 and NRG1 genes.

$625 \mathrm{a}$ and $\mathrm{b}$. The DNA methylation levels of SATB2 (a) and NRG1 (b) genes are shown in dot plots. The

626 vertical axis indicates the DNA methylation levels in the MED12m-positive uterine fibroids $(\mathrm{n}=9)$, -

627 negative uterine fibroids $(\mathrm{n}=12)$, and the corresponding myometrium. The DNA methylation levels were

628 examined by COBRA and range from 0 to $100 \%$. c and d. The expression levels of SATB2 (c) and NRG1 
629 (d) genes in the MED12m-positive uterine fibroids, -negative uterine fibroids, and the myometrium analyzed

630 by qRT-PCR are shown in dot plots. The expression levels are corrected for myometrium expression as 1. 
Table 1. COGs groups detected by WGCNA and the numbers of COGs groups with significant KEGG pathways and GO terms.

\begin{tabular}{|c|c|c|c|c|}
\hline hes & $\begin{array}{l}\text { genes assigned } \\
\text { to COGs groups }\end{array}$ & $\begin{array}{l}\text { genes without } \\
\text { assignement to } \\
\text { COGs groups }\end{array}$ & $\begin{array}{c}\text { Number of } \\
\text { detected COGs } \\
\text { groups }\end{array}$ & $\begin{array}{l}\text { Number COGs } \\
\text { groups with } \\
\text { significant GO }\end{array}$ \\
\hline
\end{tabular}

\begin{tabular}{|c|c|c|c|c|c|}
\hline MED12m-positive & 19860 & 19859 & 1 & 26 & 3 \\
\hline MED12m-negative & 19860 & 16183 & 3677 & 14 & 5 \\
\hline
\end{tabular}


Table 2. Activated signalings of cell proliferation and anti-apoptosis in differentially expressed genes in uterine fibroids.

\begin{tabular}{|c|c|c|}
\hline \multirow{2}{*}{\multicolumn{3}{|c|}{$\begin{array}{l}\text { IPA pathway }(\mathrm{p}<0.05) \\
\text { Cell proliferation }\end{array}$}} \\
\hline & & \\
\hline \multicolumn{3}{|l|}{ Wnt $/ \beta$-catenin signaling } \\
\hline \multicolumn{3}{|l|}{ HIF $1 \alpha$ - signaling } \\
\hline \multicolumn{3}{|l|}{ mTOR signaling } \\
\hline \multicolumn{3}{|l|}{ PI3K/AKT signaling } \\
\hline \multicolumn{3}{|l|}{ p70S6K signaling } \\
\hline \multicolumn{3}{|l|}{ STAT3 siganling } \\
\hline \multicolumn{3}{|l|}{ RANK signaling in osteoclasts } \\
\hline \multicolumn{3}{|l|}{ PPAR $\alpha / \mathrm{RXT} \alpha$ activation } \\
\hline \multicolumn{3}{|l|}{$\mathrm{NF}-\kappa \mathrm{B}$ signaling } \\
\hline \multicolumn{3}{|l|}{ RAR activation } \\
\hline \multicolumn{3}{|l|}{ PXR/RXR activation } \\
\hline \multicolumn{3}{|l|}{ HER-2/ErbB signaling } \\
\hline \multicolumn{3}{|l|}{ ERK/MAPK siganling } \\
\hline \multicolumn{3}{|l|}{ LPS-stimulated MAPK signaling } \\
\hline \multicolumn{3}{|l|}{ Anti-apoptosis } \\
\hline \multicolumn{3}{|l|}{ 14-3-3mediated signaling } \\
\hline \multicolumn{3}{|l|}{ PI3K/AKT signaling } \\
\hline \multicolumn{3}{|l|}{ p70S6K signaling } \\
\hline \multicolumn{3}{|l|}{ PAK signaling } \\
\hline IGF-1 signaling & & \\
\hline
\end{tabular}

Differentially expressed genes compared to the myometrium (the DEGs) in the MED12m-positive and -negative uterine fibroids were applied to KEGG pathway analysis in IPA, respectively. Detected pathways with $\mathrm{p}<0.05$ were considered significant enrichment. Activated signaling pathways related to cell proliferation and anti-apoptosis were indicated. 


\section{Figures}

a
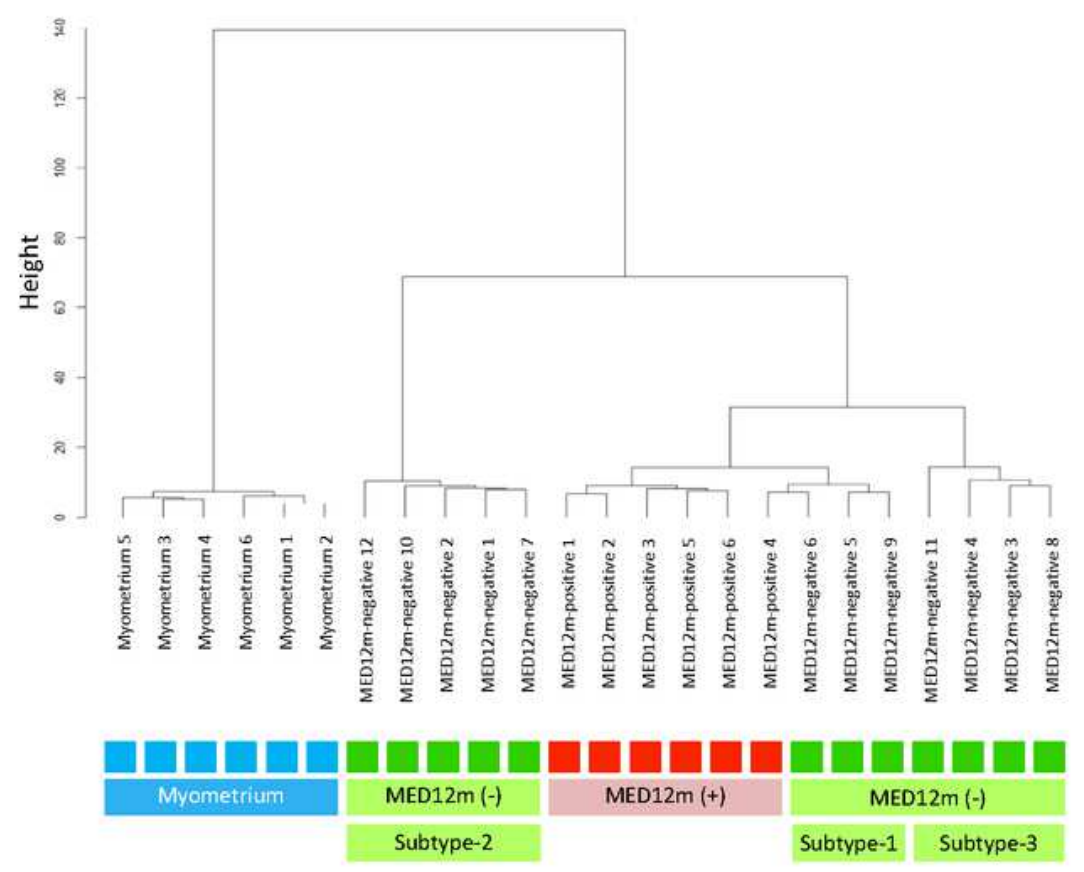

b
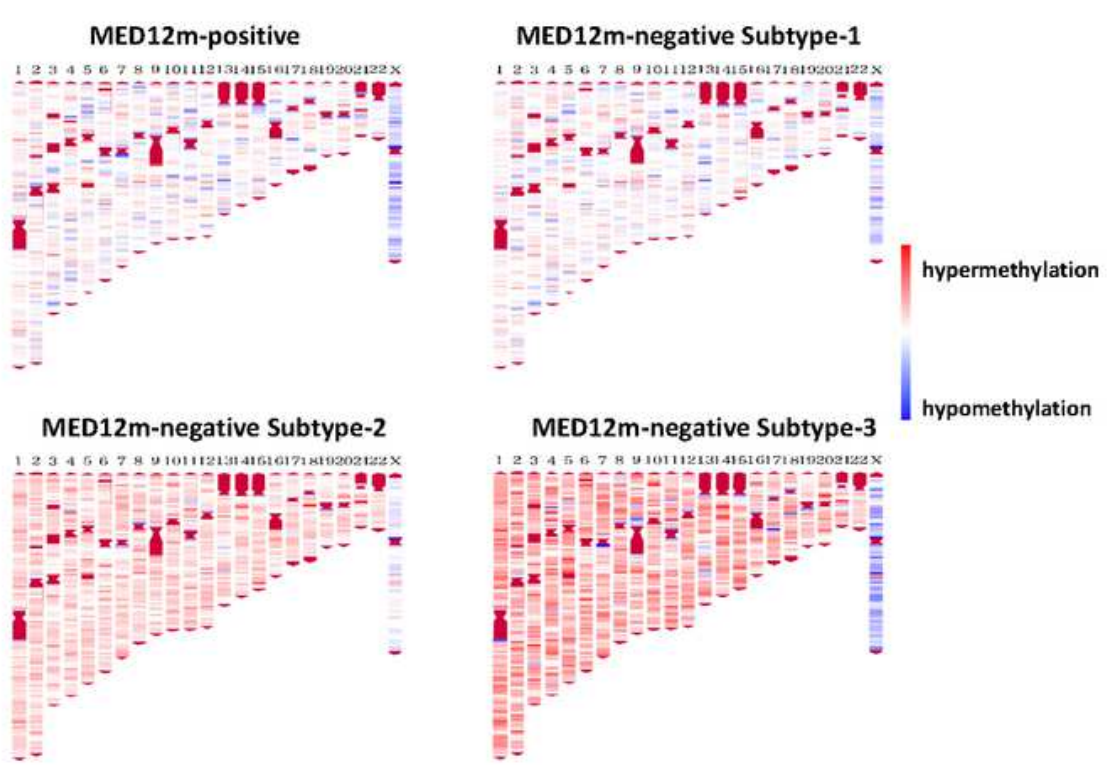

Figure 1

DNA methylation profiling of the MED12m-positive and -negative uterine fibroids, and myometrium. a. DNA methylation profiles of the MED12m-positive and -negative uterine fibroids, and myometrium were compared using hierarchical clustering analyses. Distances of DNA methylation pattern are indicated as 
height. Each color indicates the myometrium (light blue), the MED12m-positive uterine fibroids (red), and the MED12m-negative uterine fibroids (green). The MED12m-negative uterine fibroids were further classified into three different clusters, Subtype-1, Subtye-2, and Subtype-3. b. Chromosomal distribution of hyper- or hypomethylated CpGs in the MED12m-positive and -negative uterine fibroids (Subtype-1, -2, and -3 ) compared to the myometrium are shown. The locations of $\mathrm{CpG}$ sites, which have $p<0.05$ and beta value difference $>0.2$ compared to the myometrium, are indicated with red (hypermethylated $\mathrm{CpGs}$ ) or blue (hypomethylated CpGs). Autosomal and sex chromosome numbers are shown on the top.

a

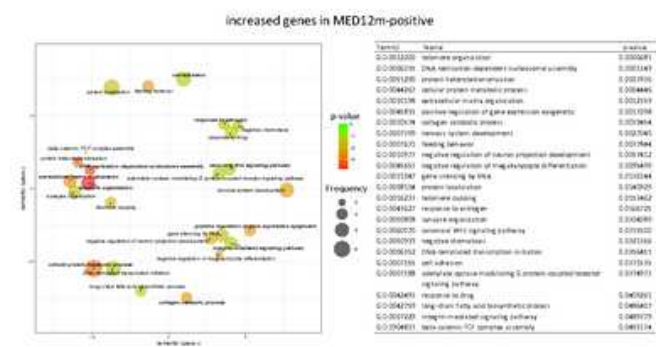

b

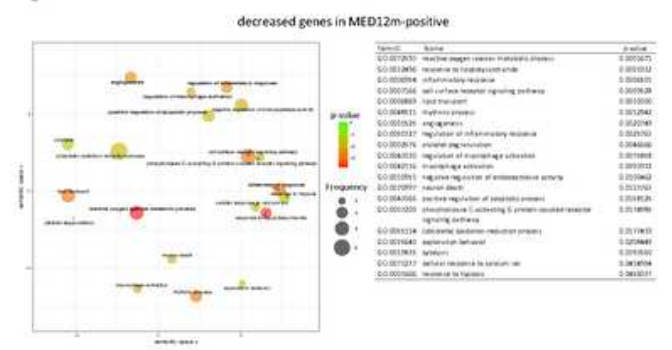

Figure 2 (continued)

c

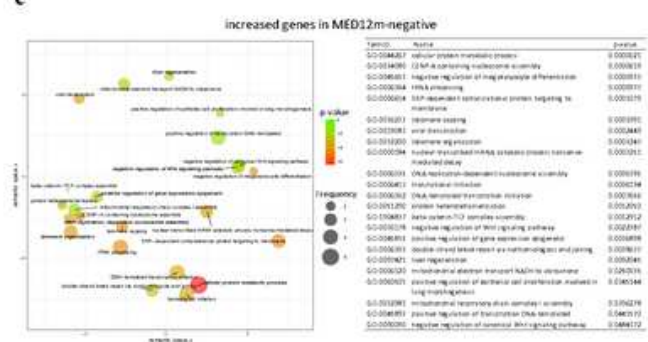

d

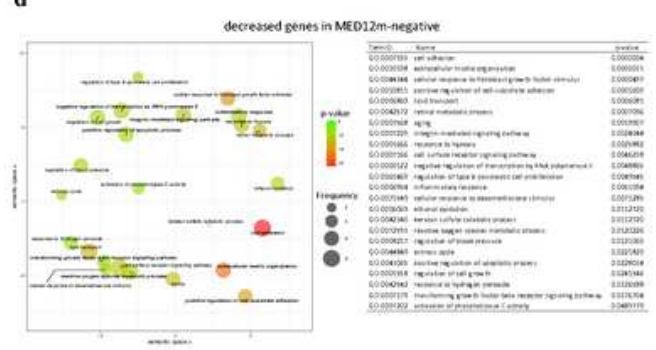

Figure 2 (continued)

e

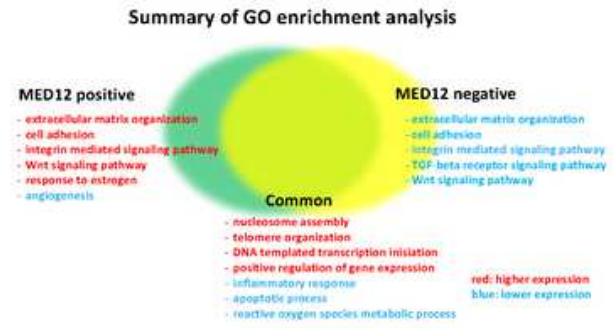




\section{Figure 2}

The scatterplot of GO terms in DEGs. The plots and tables show the GO terms after the redundancy reduction in the MED12m-positive-increased (a), -decreased (b), the MED12m-negative-increased (c), and decreased (d) DEGs. The colors indicate the log10(p-value) of the summarized GO terms. The size of the circle indicates the frequency of the $\mathrm{GO}$ term in the underlying $\mathrm{GO}$ database. The circles of more general terms are plotted larger. The color and the size of circles are plotted according to the default setting of REVIGO 39. e. Summary of GO analysis in the MED12m-positive and -negative uterine fibroids. The specific terms to the MED12m-positive uterine fibroids, the MED12m-591 negative uterine fibroids, and commonly detected terms are shown. Red and blue mean higher and lower expression compared to the myometrium 

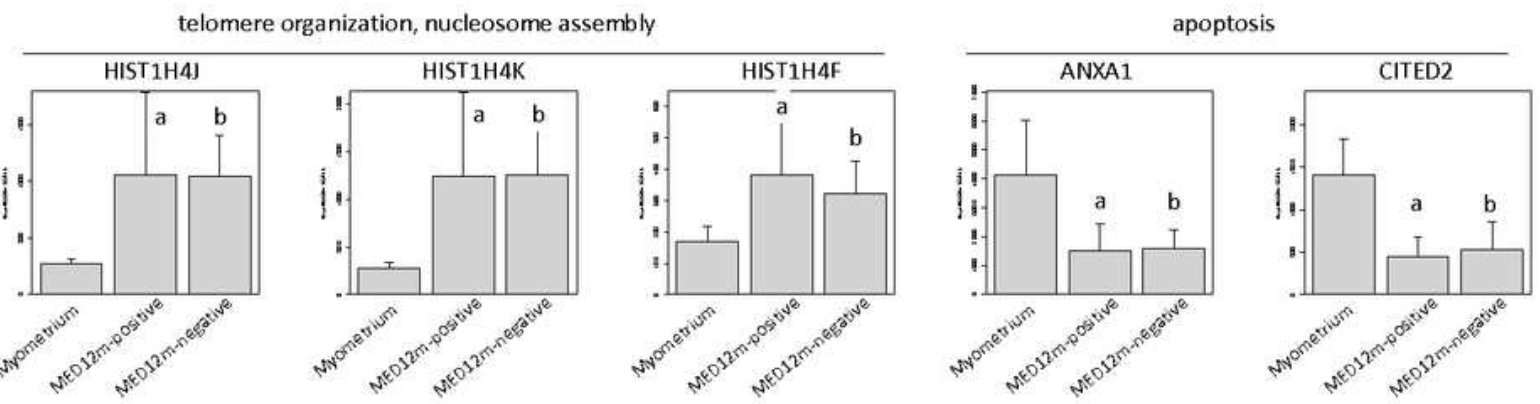

b
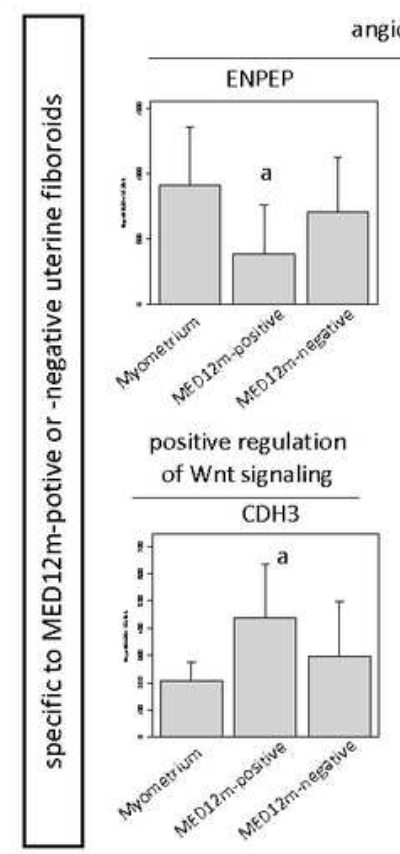

niogenesis

inflammarory response

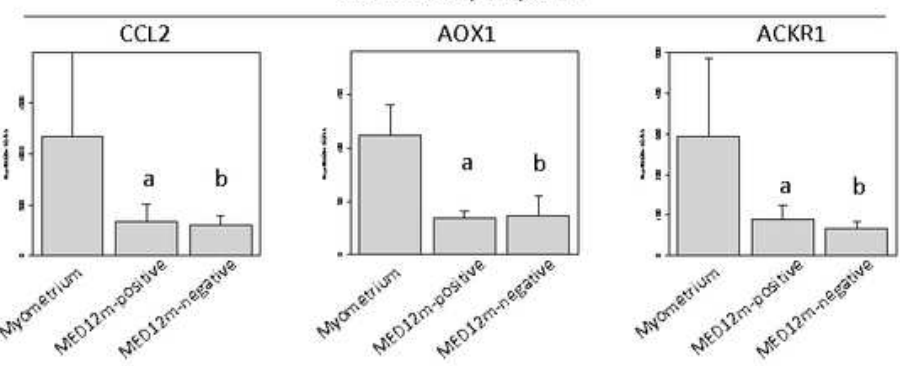

positive regulation

of Wnt signaling
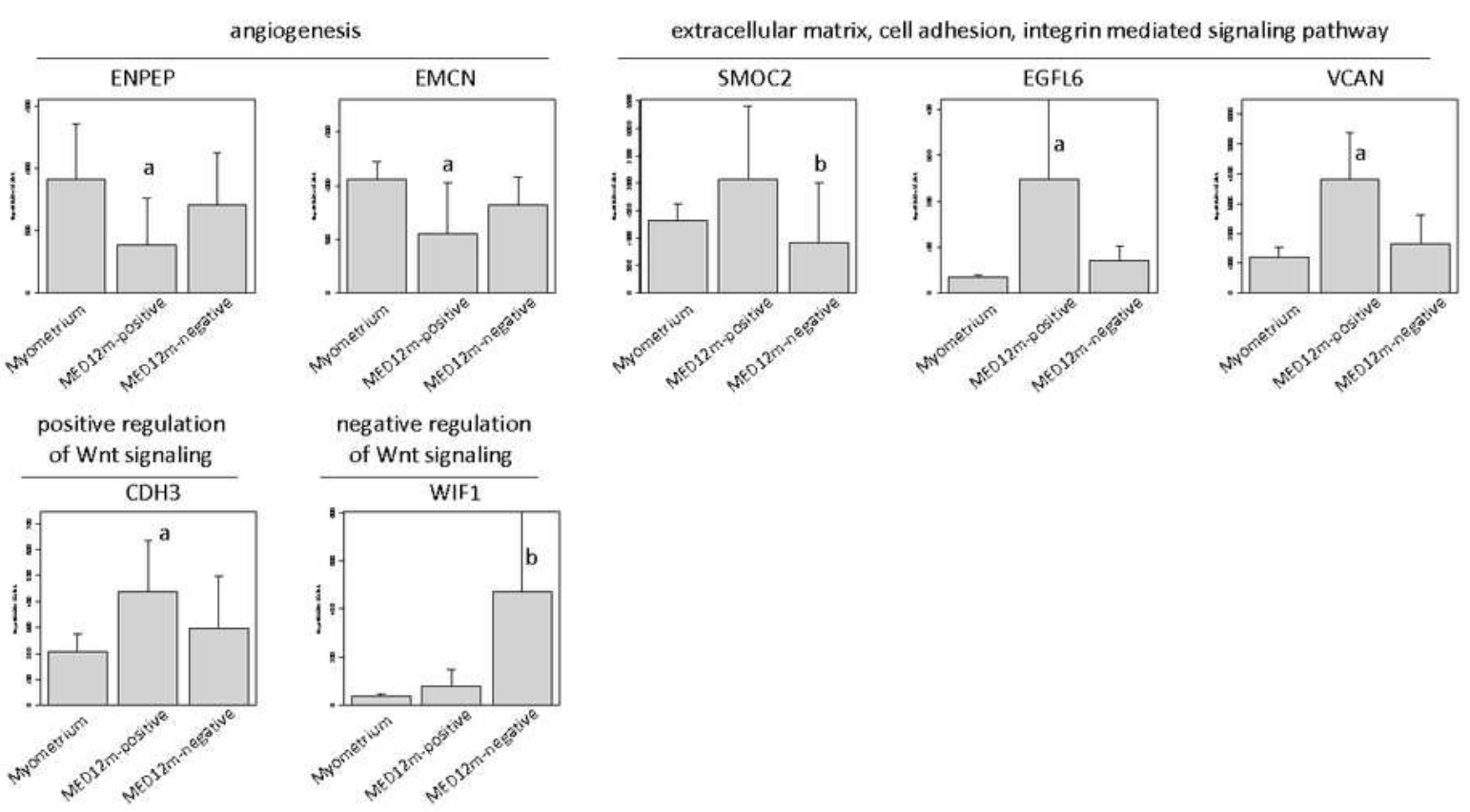

negative regulation

of Wnt signaling

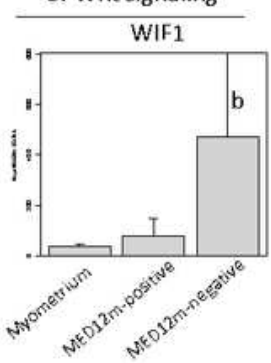

\section{Figure 3}

Expression levels of representative genes in the detected biophysical processes. Representative genes in commonly (a) or oppositely (b) activated/deactivated processes between the MED12m-positive and negative uterine fibroids were indicated. $a, p<0.01$ (myometrium $(n=6)$ vs. MED12m-positive uterine fibroids $(n=6)$ ). $b, p<0.01$ (myometrium ( $n=6)$ vs. MED12m-negative uterine fibroids $(n=9)$ ). 


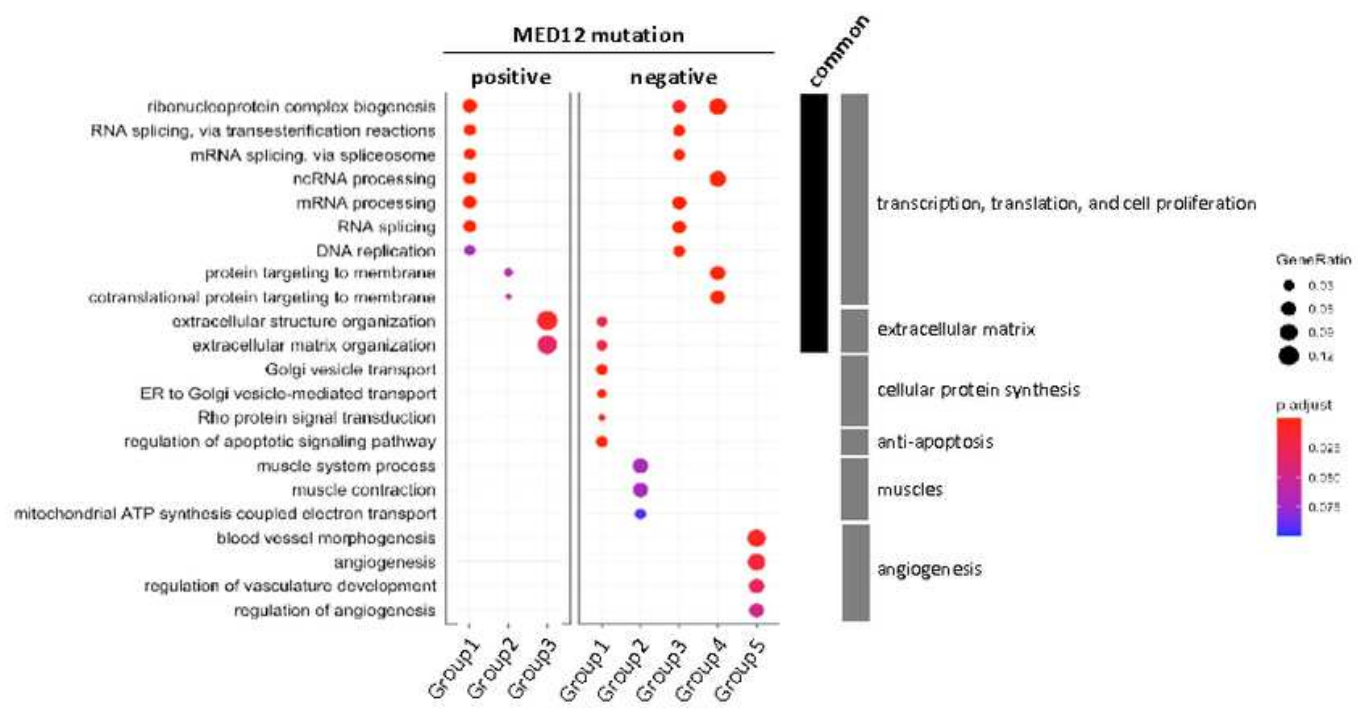

Figure 4

Enriched GO terms identified by weighted gene co-expression network analysis (WGCNA) in the MED12mpositive and -negative uterine fibroids. Twenty-six and 14 COGs groups in the MED12m-positive and negative uterine fibroids, respectively, were introduced into the KEGG pathway and GO enrichment analyses. Three and five COGs groups in the MED12m-positive and -negative uterine fibroids were significantly enriched with GO terms. The other 23 and 9 COGs groups in the MED12m-positive and negative uterine fibroids, respectively, were not significantly enriched with $\mathrm{GO}$ terms. The ratio of the number of identified genes to all genes in each term is shown as "geneRatio". P-values were adjusted with the $\mathrm{BH}$ method by clusterProfiler 36 and indicated with colors. 


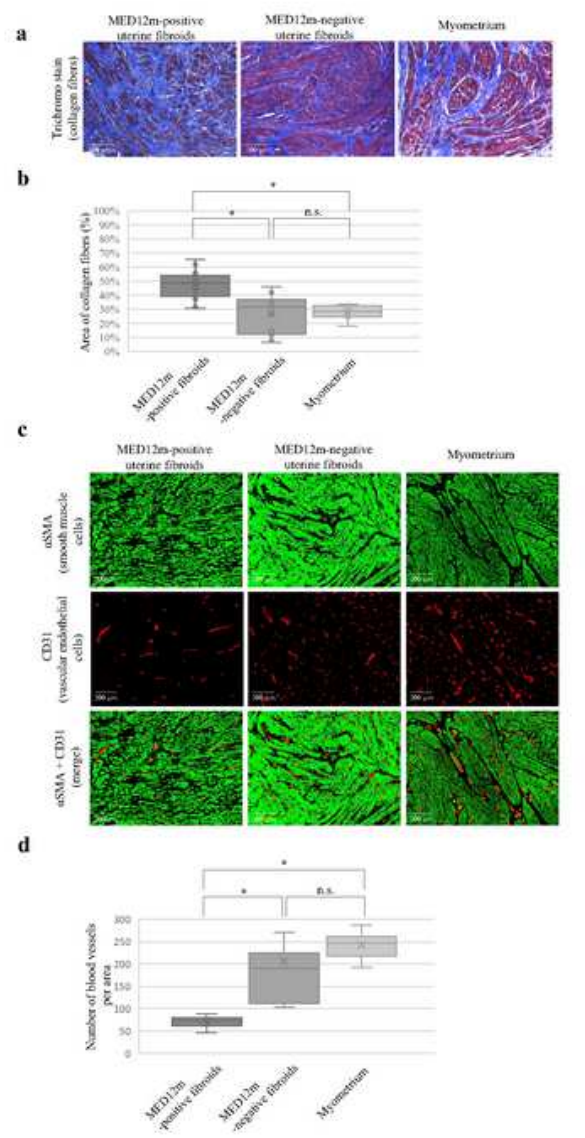

Figure 5 (continued)

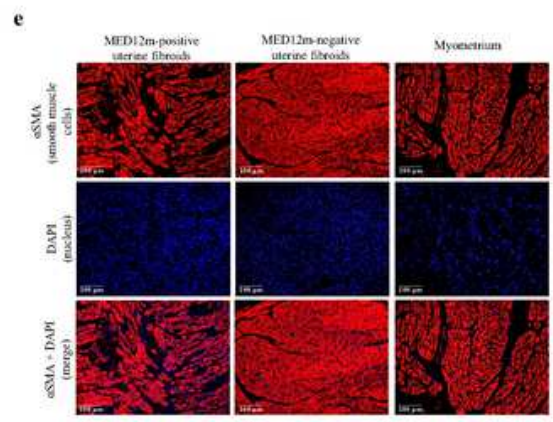

f

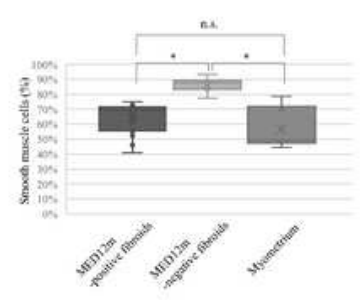

Figure 5

Histological examination in the uterine fibroids and myometrium. a. Immunofluorescent staining for collagen fibers in the MED12m-positive, -negative uterine fibroids, and myometrium. Collagen fibers are detected as blue by a trichrome staining kit (TRM-1, ScyTec Laboratories inc). b. Boxplots show the occupation rate of collagen fiber. The collagen fiber area was quantified by Image J. The percentage per view field was calculated on 15 randomly chosen areas at x200 magnification, and average percentages 
were indicated in each tissue section. *, p < 0.05. c. Immunofluorescent staining for smooth muscle cells (aSMA, green) and vascular endothelial cells (CD31, red) in the MED12m-positive, -negative uterine fibroids, and myometrium. d. Boxplots show the number of blood vessels, which was counted by Image J. The number per view field was calculated on 5 randomly chosen areas at $x 100$ magnification, and the average numbers were indicated in each tissue section. *, $p<0.05$. e. Immunofluorescent staining for smooth muscle cells (aSMA, red) and nucleus (DAPI, blue) in the MED12m-positive, -negative uterine fibroids, and myometrium. The cells stained with aSMA were considered as smooth muscle cells, whereas the cells that were not stained with aSMA was considered as non-smooth muscle cells. $f$. Boxplots show the percentage of the smooth muscle cells in the smooth and non-smooth muscle cells. The number per view field was calculated on 5 randomly chosen areas at x200 magnification, and the average numbers were indicated in each tissue section. ${ }^{*}, p<0.05$. 
a
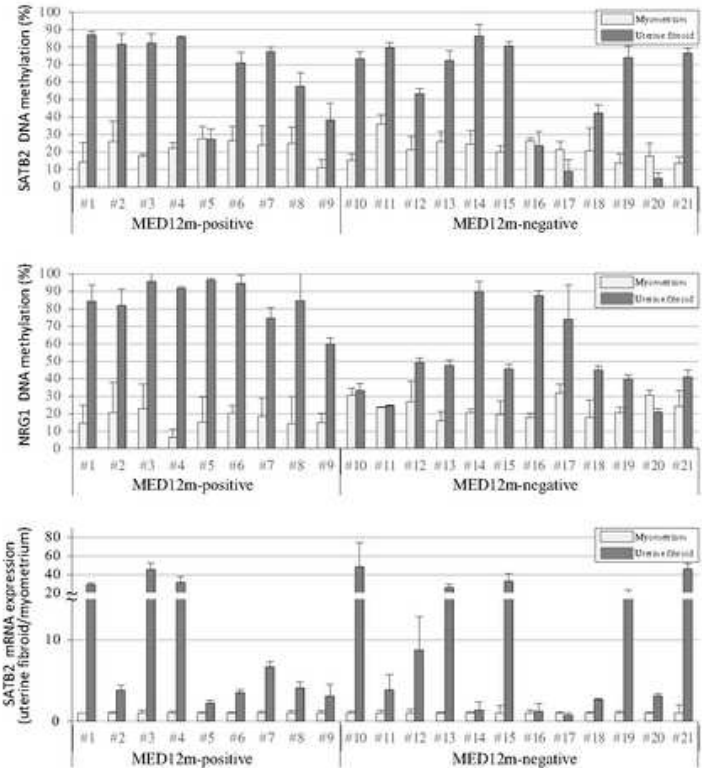

d
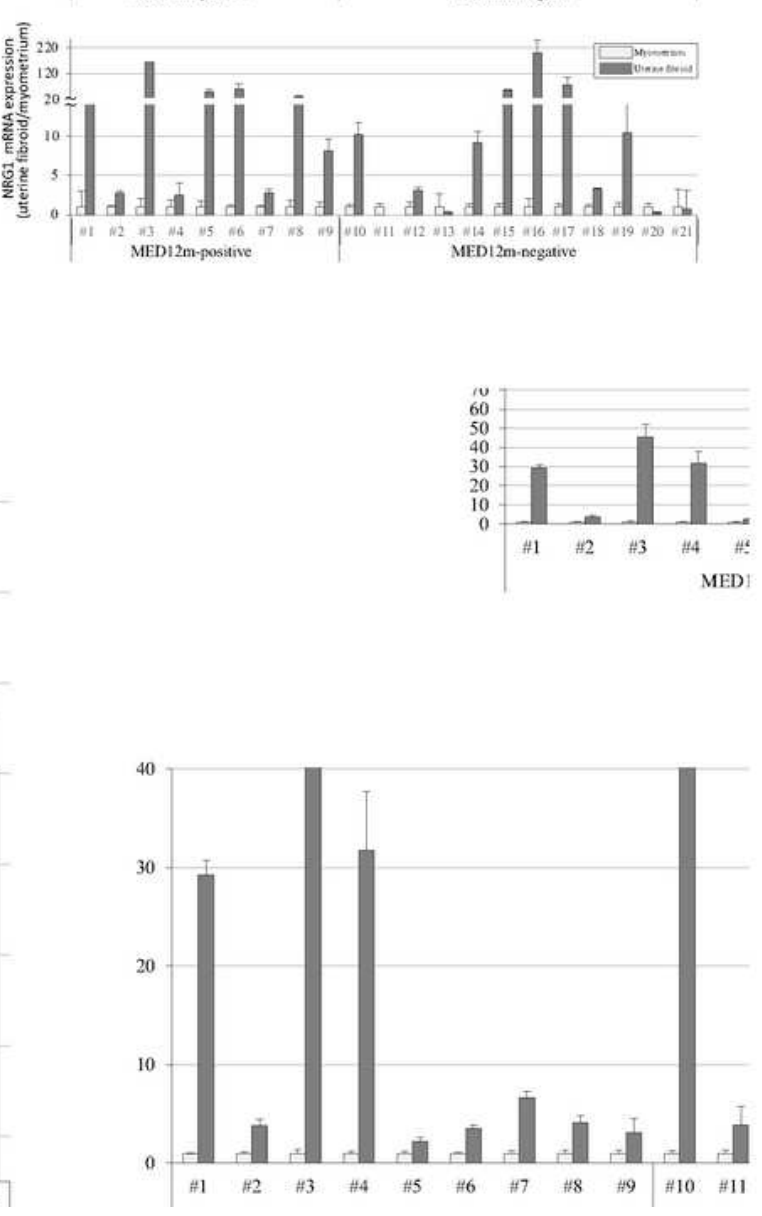

Figure 6

DNA methylation and mRNA expression statuses of SATB2 and NRG1 genes. $a$ and $b$. The DNA methylation levels of SATB2 (a) and NRG1 (b) genes are shown in dot plots. The vertical axis indicates the DNA methylation levels in the MED12m-positive uterine fibroids $(n=9)$, - negative uterine fibroids $(n=$ 12 ), and the corresponding myometrium. The DNA methylation levels were examined by COBRA and range from 0 to $100 \%$. c and d. The expression levels of SATB2 (c) and NRG1 (d) genes in the MED12m- 
positive uterine fibroids, -negative uterine fibroids, and the myometrium analyzed by qRT-PCR are shown in dot plots. The expression levels are corrected for myometrium expression as 1.

\section{Supplementary Files}

This is a list of supplementary files associated with this preprint. Click to download.

- SupplementalMaterials.pdf 